Received: 2019.03.03 Accepted: 2019.03 .27 Published: 2019.07.01

\title{
A Case of Systemic Lupus Erythematosus Presenting as Autoimmune Myelofibrosis
}

Authors' Contribution:

Study Design A

Data Collection B

Statistical Analysis C

Data Interpretation D

Manuscript Preparation E

Literature Search F

Funds Collection G
EF 1 Ikechukwu Mbonu

E 1 Christoph Sossou

E 1 Christopher B. Nnaoma

ADE 2 Xinlai Sun

D 3 Lori Schleicher

D 4 Wen Xiong
1 Department of Internal Medicine, Newark Beth Israel Medical Center, Newark, NJ, U.S.A.

2 Department of Pathology, Immunology and Laboratory Medicine, Rutgers University, New Jersey Medical School, Newark, NJ, U.S.A.

3 Department of Hematology and Oncology, Newark Beth Israel Medical Center, Newark, NJ, U.S.A.

4 Department of Rheumatology, Newark Beth Israel Medical Center, Newark, NJ, U.S.A.
Corresponding Author: Conflict of interest:

\begin{tabular}{|c|c|}
\hline Patient: & Female, 20 \\
\hline Final Diagnosis: & Systemic lupus erythematosus (SLE) \\
\hline Symptoms: & Anemia $\bullet$ fatigue $\bullet$ weakness \\
\hline Medication: & - \\
\hline nical Procedure: & - \\
\hline Specialty: & Rheumatology \\
\hline Objective: & Rare co-existance of disease or pathology \\
\hline Background: & $\begin{array}{l}\text { Systemic lupus erythematosus (SLE) is characterized by multiorgan involvement and presence of autoantibodies. } \\
\text { SLE has a broad range of presentations and manifestations, and as such, its course and organ involvement are } \\
\text { unpredictable. The disease results from the interaction of genes, environment, and random effects combining } \\
\text { to lead to a loss of tolerance to self-antigens and active autoimmunity. Autoimmune myelofibrosis is a type of } \\
\text { non-malignant bone marrow fibrosis that occurs in the presence of systemic autoimmune disease. Cytopenias } \\
\text { such as anemia, leukopenia, and thrombocyotopenia are common manifestations of SLE; however, myelofibro- } \\
\text { sis is a less common and far less recognized complication of SLE. }\end{array}$ \\
\hline Case Report: & $\begin{array}{l}\text { We report a case of a young African American female who presented with severe anemia and leukopenia, } \\
\text { subsequently diagnosed with myelofibrosis and then eventually SLE. The identification of myelofibrosis in SLE } \\
\text { is critical as it can be a devastating condition when untreated. Fortunately, autoimmune myelofibrosis in SLE } \\
\text { is reversible with treatment of the underlying condition. }\end{array}$ \\
\hline Conclusions: & $\begin{array}{l}\text { Autoimmune myelofibrosis is a rare complication of SLE. Autoimmune myelofibrosis could be the first and } \\
\text { only presenting feature of SLE. It is sensible to recognize this relationship, as prompt diagnosis and treatment } \\
\text { is crucial. Corticosteroids have been shown to be useful in treating both SLE and the associated autoimmune } \\
\text { myelofibrosis. }\end{array}$ \\
\hline
\end{tabular}

MeSH Keywords:

Anemia • Fibrosis • Hematology • Lupus Erythematosus, Systemic • Primary Myelofibrosis • Rheumatology

Full-text PDF: https://www.amjcaserep.com/abstract/index/idArt/916001

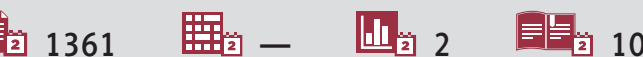




\section{Background}

Hematological abnormalities such as leukopenia, lymphopenia, thrombocytopenia, and hemolytic anemia are common findings in the course of systemic lupus erythematosus (SLE), and they are indeed part of the classification criteria of SLE [1]. Although a range of cytopenias can be commonly seen in SLE, autoimmune myelofibrosis (AIMF) is rarely seen. It was noted in a cross-sectional study that myelofibrosis is an uncommon finding among patients with SLE presenting with cytopenias [2]. AIMF and primary immune fibrosis are 2 similar but distinct entities that need distinguishing. In the few reported cases of AIMF in SLE, AIMF tends to present either concurrently with the diagnosis of SLE or after the diagnosis of SLE has been made. We present a case of AIMF presenting prior to the diagnosis of SLE in a young female patient.

\section{Case Report}

A 20-year-old African American female with no prior medical history presented to the hospital with worsening fatigue, dyspnea on exertion, and palpitations of over 1 month occurrence. The patient reported difficulty climbing more than 1 flight of stairs due to shortness of breath. She also noted slightly heavier than usual menstrual periods. She denied any history of anemia, easy bruising or bleeding, yellowing of skin, rashes, or prior blood transfusions. Review of systems was positive for fever several weeks before presentation but otherwise negative. The patient was not on any medications, had no prior surgeries, and had no known allergies. The patient denied using alcohol, tobacco, or any illicit substances.

On physical examination, her blood pressure was $116 / 79 \mathrm{mmHg}$, heart rate was 114 beats per minute, respiratory rate was 18 breaths per minute, temperature was $36.9^{\circ} \mathrm{C}\left(98.4^{\circ} \mathrm{F}\right)$, weight was $91.8 \mathrm{~kg}$, height was $163 \mathrm{~cm}$, and body mass index was $34.55 \mathrm{~kg} / \mathrm{m}^{2}$. The patient was alert, oriented, and in no acute distress. The rest of the clinical examination was insignificant except for tachycardia on cardiovascular examination and pale conjunctiva on eye examination.

Laboratory data on admission included hemoglobin of $5.2 \mathrm{~g} / \mathrm{dL}$, hematocrit $14.1 \%$, red blood cell (RBC) count $1.74 \times 10^{6} \mathrm{mcL}$, platelet count $404 \times 10^{3}$, white blood cell count $6.2 \times 10^{3}$, and reticulocyte count $0.1 \%$. Lactate dehydrogenase (LDH) was elevated at 235 unit/L, alanine aminotransferase (ALT), aspartate aminotransferase (AST), and alkaline phosphate were all within normal limits. The patient was admitted to the inpatient ward where she was transfused with 3 units of packed RBCs and her hemoglobin increased to $7.5 \mathrm{~g} / \mathrm{dL}$. A peripheral smear showed poikilocytosis, microcytosis, and thrombocytosis. The patient's clinical symptoms improved and she was

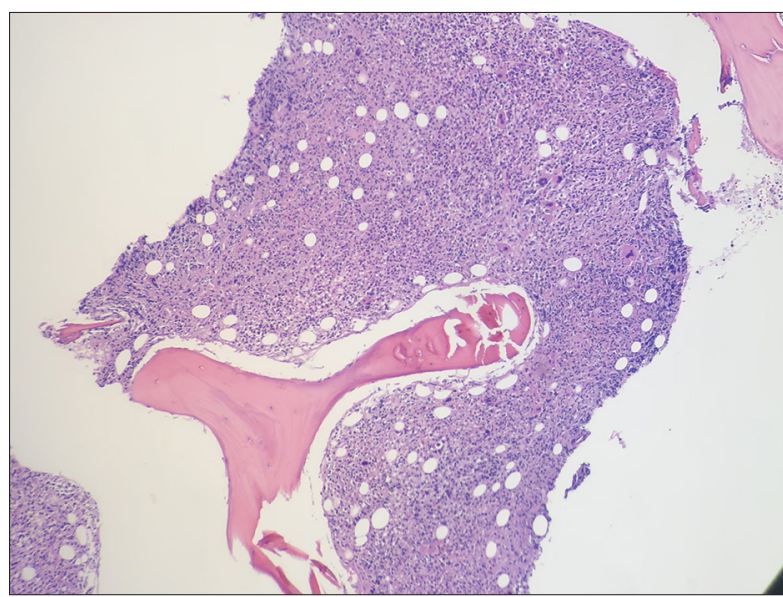

Figure 1. Bone marrow core biopsy (hematoxylin and eosin stain, 100x).

discharged and instructed to follow-up as an outpatient for further workup.

The patient received a workup in the outpatient clinic and underwent a bone marrow biopsy. The pathology report was significant for moderately increased reticulin fibrosis and mildly increased megakaryocytes in the marrow (Figure 1). These findings, in addition to the lack of bone marrow aspirates, suggested a diagnosis of myelofibrosis.

The patient returned to the Emergency Department for complaints of diffuse bone pain and fevers. The patient also complained of a lack of appetite and endorsed a 9 pound weight loss. She denied any skin rash, swollen joints, oral ulcers, or chest pain. On physical examination, the patient had multiple lymph nodes of varying sizes, non-tender in anterior and posterior cervical chains and submandibular and supraclavicular regions. Complete blood count at that time showed white blood cell (WBC) count of $3.4 \times 10^{3} \mathrm{mcL}$, RBC count of $3.09 \times 10^{6} \mathrm{mcL}$, hemoglobin of $9.2 \mathrm{~g} / \mathrm{dL}$ with a hematocrit of $27 \%$, and platelet count of $236 \times 10^{3} \mathrm{mcL}$. Mean platelet volume (MPV) was $8.6 \mathrm{fL}$, mean corpuscular volume (MCV) was $87.5 \mathrm{fL}$, mean corpuscular hemoglobin $(\mathrm{MCH})$ was $29.8 \mathrm{pg}$, mean corpuscular hemoglobin concentration (MCHC) was $34 \mathrm{~g} / \mathrm{dL}$, and red cell distribution width (RDW) was $15.7 \%$. Sedimentation rate was elevated, $>145 \mathrm{~mm} /$ hour and ferritin level was also elevated at 710. The patient was admitted for anemia and intractable bone pain. Rheumatology service was consulted and after clinical assessment, testing was done for antinuclear antibody (ANA), C3/C4, dsDNA, RF/CCP, ss-a, ss-b, and anti-Smith/RNP. A right groin excisional lymph node biopsy was done which showed mild follicular hyperplasia and paracortical expansion which favored reactive lymph node. Immunostains supported the diagnosis of reactive lymph node. All mutational tests for JAK2, MPL, CALR, and BCR-ABL came back negative. Rheumatology workup revealed ANA positive, dsDNA antibody positive (15), 
rheumatoid factor positive ( $>1000)$, anti-Smith antibody positive, and SSA antibody positive. ANA titer was reported at 1: 2560 with a homogeneous ANA pattern. C4 complement level was low at $<3 \mathrm{mg} / \mathrm{dL}$ and C3 complement level was low at $<63 \mathrm{mg} / \mathrm{dL}$. The patient was started on intravenous SoluMedrol and then discharged on a steroid taper and Plaquenil. The patient was instructed to follow-up with Rheumatology service and her primary physician as an outpatient.

\section{Discussion}

Systemic lupus erythematosus (SLE) is characterized by multiorgan involvement and the presence of autoantibodies. Patients with SLE have abnormalities in how dying cells are handled by the immune system [3]. It is a basic failure of the mechanisms that maintain self-tolerance [4]. The nuclear material of dying cells are not properly cleared, producing an immune response, and promoting upregulation of autoreactive $T$ and $B$ cells and autoantibodies directed against nuclear antigens. The end result is a cycle of antigen release and immune cell activation causing the production of high-affinity autoantibodies [5]. About $90 \%$ of patients with SLE are women, with the risk in disease increasing dramatically with the appearance of female sex hormones.

The disease is more common and more severe often times among women of African American, Chinese, and Hispanic background. It is thought that single gene mutations causing deficiencies of the complement components $\mathrm{C} 1 \mathrm{q}, \mathrm{C} 2$, or $\mathrm{C} 4$ can promote SLE, possibly by impairing clearance of immune complexes and apoptotic cell debris [3].

Patients with SLE commonly have a blood dyscrasia such as anemia ( $80 \%$ of SLE patients), leukopenia (50\% of SLE patients), or thrombocytopenia (30\% to $50 \%$ of SLE patients); but SLE rarely involves the bone marrow to the extent of fibrosis. Myelofibrosis or bone marrow fibrosis is a common feature of hematologic malignancies but it is found rarely in autoimmune disorders. Autoimmune myelofibrosis (AIMF) is a type of nonmalignant fibrosis that occurs in the presence of systemic autoimmune disease [6]. Primary myelofibrosis on the other hand occurs in the absence of autoimmune disease. It is important to differentiate between AIMF and primary myelofibrosis because their treatments differ markedly [7]. The AIMF pattern typically includes reticulin and collagen fibrosis, as well as different levels of cellularity in the bone marrow (Figure 2). This includes, but is not limited to the absence of abnormal megakaryotic, erythroid, and myeloid cells, the peripheral blood disorders with teardrop poikilocytosis and leukoerythroblastosis, and bone marrow lymphoid aggregates and the existence of autoantibodies [8].

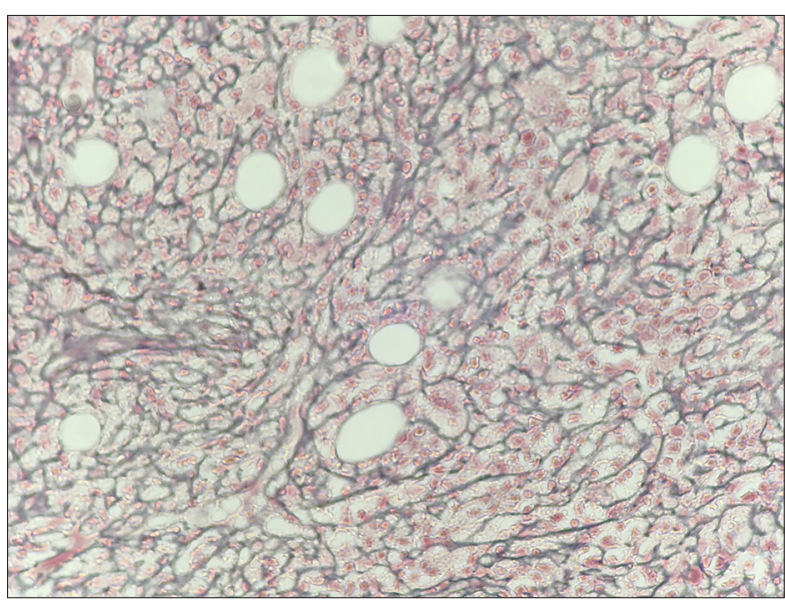

Figure 2. Bone marrow core biopsy (reticulin stain, 400x).

The pathogenesis of AIMF is not completely understood, but appears to be a relative nonspecific response of fibroblasts to underlying cellular abnormalities [3]. Increased reticulin is the result of fibroblast proliferation and increased collagen synthesis or altered collagen turnover that appears to be due to decreased collagenase release [9]. Primary myelofibrosis is diagnosed usually in the sixth decade of life (median age is 66 years) and more commonly in males. AIMF occurring with SLE is usually diagnosed earlier (median age is 29 years) and is not very common in males (ratio is 1: 9) [8]. Autoimmune fibrosis often occurs in patients with preceding diagnosis of SLE, however, in rare cases, such as in our case, autoimmune fibrosis can be a presenting condition of SLE.

It is possible that AIMF is underestimated and underreported in cases of SLE [10]. This could be due to the fact that autoimmune fibrosis and SLE share some clinical features, which sometimes overlap. It is important to evaluate for autoimmune fibrosis and even perform a bone marrow biopsy in SLE patients presenting with cytopenias. This is crucial as bone marrow fibrosis in SLE is responsive to steroids and other immunosuppressive drugs and has a favorable outcome [8], whereas primary myelofibrosis is not responsive to conventional SLE treatment and has a less favorable outcome.

\section{Conclusions}

SLE is an autoimmune condition with many presentations and complications. Hematological manifestations of SLE are quite common, however, AIMF is rare and not a common presentation of SLE.

\section{Conflicts of interests}

None. 


\section{References:}

1. Dondu U, Hava U, Cengiz K: A rare cause of cytopenia in a patient with systemic lupus erythematosus: Autoimmune myelofibrosis. Eur J Rheumatol, 2017; 4: 76-78

2. Wanitpongpun C, Teawtrakul N, Mahakkanukrauh A et al: Bone marrow abnormalities in systemic lupus erythematosus with peripheral cytopenia. Clin Exp Rheumatol, 2012; 30: 825-29

3. Maidhof W, Hilas O: Lupus: An overview of the disease and management options. P T, 2012; 37(4): 240-49

4. Silver EM, Ochoa W: Glucocorticoid-induced myopathy in a patient with systemic lupus erythematosus (SLE): A case report and review of the literature. Am J Case Rep, 2018; 19: 277-83

5. Vinay K, Abbas AK, Fausto $\mathrm{N}$ et al: Robbins and cotran pathologic basis of disease. $8^{\text {th }}$ ed. Philadelphia: Elsevier Saunders, 2010; 219-18
6. Gauiran D, Cheng P, Pagaduan C, Santos M: Autoimmune-associated hemophagocytosis and myelofibrosis in a newly diagnosed lupus patient: Case report and literature review. Case Rep Hematol, 2019; 2019: 3879148

7. Pullarkat V, Bass RD, Gong JZ et al: Primary autoimmune myelofibrosis: Definition of a distinct clinicopathologic syndrome. Am J Hematol, 2002; 72(1): 8-12

8. Chalayer É, Ffrench M, Cathébras P: Bone marrow fibrosis as a feature of systemic lupus erythematosus: A case report and literature review. Springerplus, 2014; 3(1): 349

9. Pundole X, Konoplev S, Oo T, Lu H: Autoimmune myelofibrosis and systemic lupus erythematosus in a middle-aged male presenting only with severe anemia. Medicine, 2015; 94(19): e741

10. Del Porto F, Tatarelli C, Proietta M: Systemic lupus erythematosus and myelofibrosis: A case report and revision of literature. Leuk Res Rep, 2018; 9: 58-64 